\title{
BMJ Open Protocol for a prospective, longitudinal cohort study on the effect of arterial disease level on the outcomes of supervised exercise in intermittent claudication: the ELECT Registry
}

\author{
Marijn ML van den Houten, ${ }^{1,2}$ Sandra CP Jansen, ${ }^{1,2}$ Anneroos Sinnige, ${ }^{1}$ \\ Lijckle van der Laan, ${ }^{3}$ Patrick WHE Vriens, ${ }^{4}$ Edith M Willigendael, ${ }^{5}$ \\ Jan-Willem HP Lardenoije, ${ }^{6}$ Jan-Willem M Elshof, ${ }^{7}$ Eline S van Hattum, ${ }^{8}$ \\ Maarten A Lijkwan, ${ }^{9}$ Ivan Nyklíček, ${ }^{10}$ Ellen V Rouwet, ${ }^{11}$ Mark JW Koelemay, ${ }^{12}$ \\ Marc RM Scheltinga, ${ }^{13}$ Joep AW Teijink ${ }^{1,2}$
}

To cite: van den Houten MML, Jansen SCP, Sinnige A, et al. Protocol for a prospective, longitudinal cohort study on the effect of arterial disease level on the outcomes of supervised exercise in intermittent claudication: the ELECT Registry. BMJ Open 2019;9:e025419. doi:10.1136/ bmjopen-2018-025419

- Prepublication history and additional material for this paper are available online. To view these files, please visit the journal online (http://dx.doi. org/10.1136/bmjopen-2018025419).

Received 17 July 2018 Revised 15 December 2018 Accepted 20 December 2018

D Check for updates

(c) Author(s) (or their employer(s)) 2019. Re-use permitted under CC BY-NC. No commercial re-use. See rights and permissions. Published by BMJ.

For numbered affiliations see end of article.

Correspondence to Dr Joep AW Teijink; joep.teijink@ catharinaziekenhuis.n

\section{ABSTRACT}

Introduction Despite guideline recommendations advocating conservative management before invasive treatment in intermittent claudication, early revascularisation remains widespread in patients with favourable anatomy. The aim of the Effect of Disease Level on Outcomes of Supervised Exercise in Intermittent Claudication Registry is to determine the effect of the location of stenosis on the outcomes of supervised exercise in patients with intermittent claudication due to peripheral arterial disease.

Methods and analysis This multicentre prospective cohort study aims to enrol 320 patients in 10 vascular centres across the Netherlands. All patients diagnosed with intermittent claudication (peripheral arterial disease: Fontaine I//Rutherford 1-3), who are considered candidates for supervised exercise therapy by their own physicians are appropriate to participate. Participants will receive standard care, meaning supervised exercise therapy first, with endovascular or open revascularisation in case of insufficient effect (at the discretion of patient and vascular surgeon). For the primary objectives, patients are grouped according to anatomical characteristics of disease (aortoiliac, femoropopliteal or multilevel disease) as apparent on the preferred imaging modality in the participating centre (either duplex, CT angiography or magnetic resonance angiography). Changes in walking performance (treadmill tests, 6 min walk test) and quality of life (QoL; Vascular QoL Questionnaire-6, WHO QoL Questionnaire-Bref) will be compared between groups, after multivariate adjustment for possible confounders. Freedom from revascularisation and major adverse cardiovascular disease events, and attainment of the treatment goal between anatomical groups will be compared using Kaplan-Meier survival curves.

Ethics and dissemination This study has been exempted from formal medical ethical approval by the Medical Research Ethics Committees United 'MEC-U' (W17.071). Results are intended for publication in peer-reviewed journals and for presentation to stakeholders nationally and internationally.
Strengths and limitations of this study

This is the first prospective, longitudinal study linking the outcomes of supervised exercise therapy in intermittent claudication to the location and extent of the underlying atherosclerotic lesions.

- Apart from clinical outcome measures such as freedom from intervention, the Effect of Disease Level on Outcomes of Supervised Exercise in Intermittent Claudication Registry includes a wide range of functional measures of walking performance and quality of life.

- Potential participants are approached in 10 vascular surgery centres across the Netherlands, ensuring a varied patient population and clinical practice reflective of standard care in the Dutch healthcare system.

- The location and extent of stenosis is determined using duplex ultrasound scanning in most participants; a non-invasive and valid tool, but prone to visualisation issues in case of bowel gas or unfavourable body habitus.

- Participants with aortoiliac disease and femoropopliteal disease are expected to differ regarding several clinical characteristics (eg, smoking status, diabetes mellitus, body mass index and ankle-brachial index) and adjustment for these variables will be performed in the analysis, but unmeasured confounding could influence the results.

Trial registration number NTR7332; Pre-results.

\section{INTRODUCTION}

Peripheral arterial disease (PAD) is a chronic condition caused by atherosclerotic narrowing and blocking of the peripheral arteries. Intermittent claudication (IC) is the most common manifestation of PAD and 
is marked by exertional discomfort in the leg muscles. These symptoms limit walking ability leading to functional disability in daily life. Treatment of IC symptoms aims at improving walking capacity and thereby health-related quality of life (QoL). ${ }^{1}$ Over the past decade, supervised exercise therapy (SET) and endovascular revascularisation (ER) have been shown to be equally effective in this regard..$^{2-8}$ As SET is the non-invasive ${ }^{9}$ and less costly ${ }^{10}$ option, current guidelines recommend SET as primary treatment in the management of patients with IC. ${ }^{1}$ Ideally, invasive treatment is saved for patients unresponsive to SET, which is the case in approximately $20 \%$ of patients after 2 years. ${ }^{11}$

Clinical practice often deviates from the guidelines, as worldwide reimbursement issues and lacking availability of adequate SET programmes hamper widespread adoption. ${ }^{12-14}$ In the Dutch healthcare system, SET is both available and reimbursed. ${ }^{15}$ Nevertheless, a significant proportion of patients receive early ER in the Netherlands. ${ }^{11}$ Some vascular professionals argue that in certain subsets of patients SET will probably fail and a lower threshold to initiate invasive treatment is warranted. Divergent reasons, oftentimes contradictory, are cited considering factors such as age, comorbidity, (vascular) medical history or personality traits. However, these claims are mostly practice-based as the current literature provides no grounds to discern a subset of patients who will be unresponsive to SET.

One of the main arguments for early revascularisation is the location and extent of the atherosclerotic lesion. Excellent patency rates and procedural results of aortoiliac ER in clinical trials prompt some clinicians to employ more liberal indications to intervene first in these patients. ${ }^{16}$ However, three randomised controlled trials (RCTs) compared SET with ER for patients with IC due to an iliac artery obstruction and found no important differences regarding walking distance or QoL. ${ }^{3517}$ Nonetheless, the idea that in a real-world setting individuals with proximal disease might experience less improvement after exercise training compared with patients with distal lesions remains widespread. Most studies examining the functional outcomes of patients after following a SET programme do not specify the anatomic distribution of disease. Greenhalgh $e t a l^{18}$ reported the outcome of SET for aortoiliac and femoropopliteal disease separately, from a trial comparing SET with ER. No formal comparison between outcomes in both anatomic groups was made and the sample size was small, but no apparent difference in effectiveness can be noted. The premise that the outcomes of SET depend on anatomic location and extent of disease is not based on empirical evidence.

Based on the available evidence an inferior effect of SET due to lesion location cannot be assumed. Therefore, the primary aim of this study is to determine the effect of the location of stenosis (femoropopliteal vs aortoiliac vs multilevel disease) on the outcomes of SET in patients with IC. To this end, the functional and clinical outcomes from 'real world' subjects treated with SET will be recorded, applying a minimal amount of subject selection criteria.

\section{Research objectives}

\section{Primary objective}

The aim of the Effect of Disease Level on Outcomes of Supervised Exercise in Intermittent Claudication (ELECT) Registry is to determine the effect of the location of stenosis on the outcomes of SET in patients with IC (PAD; Fontaine II, Rutherford 1-3), by recording the clinical outcomes from consecutive 'real world' subjects treated with SET. The primary objective is to determine the outcomes of SET in patients with aortoiliac disease compared with femoropopliteal disease with regard to the following measures:

1. The primary end point is change in maximum and functional walking distance on a standardised treadmill test after 3, 6 and 12 months of SET.

2. Change in 6-minute walk test (6MWT) performance after 3, 6 and 12 months of SET.

3. Change in Vascular QoL Questionnaire-6 (VascuQoL-6) and WHO QoL Questionnaire-Bref (WHOQoL-BREF) outcomes at 3 and 6 months, and 1, 2 and 5 years, follow-up.

4. Freedom from vascular interventions for the lower-extremities, at 6 months and 1, 2, and 5 years, follow-up.

5. Achievement of the main treatment goal, as drafted by the physical therapist and patient at the start of the SET programme, and indicated in the feedback letter after 3, 6 and 12 months of SET.

6. Freedom from major adverse cardiovascular events at 1,2 and 5 years, follow-up.

\section{Secondary objectives}

This study will also determine the outcomes of SET in patients with aortoiliac disease compared with femoropopliteal disease, multilevel disease and patients without aortoiliac and femoropopliteal disease, with regard to the above-mentioned measures.

The ELECT Registry dataset will furthermore be used to investigate the effect of SET on the overall IC population regarding the following objectives:

- To determine whether several baseline clinical characteristics and functional measures are predictive of changes in walking performance and clinical outcomes of SET.

- To determine whether specific personality traits (extraversion, neuroticism, conscientiousness, self-control, barrier self-efficacy, anxiety, depression and optimism) measured at baseline are predictive of the clinical outcomes of SET.

- To determine the change in barrier self-efficacy after 3, 6 and 12 months of SET.

\section{METHODS}

\section{Study design and setting}

The ELECT Registry is a multicentre prospective cohort study initiated from the vascular surgery department of 


\section{Box 1 Eligibility criteria.}

\section{Inclusion criteria}

- IC due to unilateral or bilateral PAD (Fontaine II, Rutherford 1-3).

- Resting $\mathrm{ABl}<0.9$ (or $\mathrm{TBl}<0.7$ ) or drop in $\mathrm{ABl}>0.15$ after exercise.

- Candidate for SET as a primary treatment, at the discretion of the treating vascular surgeon.

- Recent or planned imaging of at least the aortoiliac and femoropopliteal tract (within 6 months of SET initiation, but prior to possible vascular intervention): either colour duplex scanning or CTA or MRA.

S Signed informed consent form.

\section{Exclusion criteria}

- Advanced PAD beyond IC (ie, ischaemic rest pain and/or ulcers, Fontaine $>$ Il, Rutherford 4-6).

- High probability of non-adherence to physician's, or physical therapist's follow-up requirements (eg, due to lack of motivation or past compliance issues).

- Current participation in a concurrent trial that may confound study results.

- Vascular intervention as primary treatment, at the discretion of the treating vascular surgeon.

- Prior SET, performed in accordance with the guidelines of the Dutch Society for Physical Therapists, in the previous 12 months.

- Prior revascularisation in the lower extremities in the previous 12 months.

- Neurogenic/venous/orthopaedic claudication more dominant than arterial claudication complaints.

$\mathrm{ABI}$, ankle-brachial index; CTA, computed tomographic angiography; IC, intermittent claudication; MRA, magnetic resonance angiography; PAD, peripheral arterial disease; SET, supervised exercise therapy; TBI, toe-brachial index.

the Catharina Hospital in Eindhoven, the Netherlands. All patients diagnosed with IC (PAD; Fontaine II/Rutherford 1-3), who are considered candidates for SET by their own physicians, and meet the inclusion criteria (box 1), are eligible. Recruitment will take place in 10 vascular surgery departments throughout the Netherlands: the Catharina Hospital, Amphia Hospital, Elisabeth Twee Steden Hospital, Albert Schweitzer Hospital, Rijnstate Hospital, Medical Spectrum Twente, University Medical Centre Utrecht, Amsterdam University Medical Centre, Máxima Medical Centre and VieCuri Medical Centre. In these centres, the treating vascular surgeon will seek verbal consent from eligible patients to be approached by a research coordinator. Candidates will subsequently receive written information on the study, inviting them to participate, with two consent forms and a self-addressed envelope. These patients will be contacted by telephone by the coordinating investigator within 1 week after their visit to the vascular surgeon to establish formal agreement to participate in the study. If the patient agrees to participate, he/she will sign the consent forms and will send both forms in the self-addressed envelope to the coordinating investigator. After receiving the two consent forms, the coordinating investigator will sign both forms and return one version to the patient. To ensure adequate data collection, the participating centres are recommended to schedule the subjects' follow-up visit(s) based on the current standard of care as prescribed by the Dutch guidelines, which is at 3-6 months. At this moment, the decision to either continue conservative management, or treat invasively (endovascular or open revascularisation) is generally made.

\section{Treatment of subjects}

All patients will receive standard cardiovascular risk management by their physician; including smoking cessation advice, statin therapy and platelet inhibitors, as explicated in the multidisciplinary guidelines. ${ }^{1}$ Furthermore, patients will receive a standard regimen of SET, which entails exercise and lifestyle coaching. SET is provided by qualified physical therapists according to usual practice (specified in the physical therapy guidelines). ${ }^{19}$ All therapists are affiliated with ClaudicatioNet, a Dutch network of physical therapists specialised in SET with lifestyle guidance. This guarantees uniform quality of care through mandatory training courses in practice guidelines, motivational interviewing skills and other IC-relevant topics. ${ }^{15}$ A typical SET programme contains up to 37 individual sessions, spanning 3-12 months. A session consists of $30 \mathrm{~min}$ of treadmill-based or track-based exercise. The initial workload of the treadmill is set to a speed and grade that elicits claudication symptoms within 3-5 min. Patients are asked to continue to walk at this workload until they experience claudication of moderate severity. A brief period of rest permits symptoms to resolve. An exercise-rest-exercise cycle is repeated several times. Such a programme requires intense monitoring of patients aimed at increasing workload by adjusting treadmill grade or speed (or both). ${ }^{15} 19$ This will generally be performed at least three times a week in the first 4 weeks and one to two times a week for the next 8 weeks. ${ }^{15}$ After that, there is a maintenance phase during which SET will be given for one to two times a month. As stated in their guidelines, the physical therapist records several outcome measurements at 3, 6, 9 and 12 months follow-up. ${ }^{19}$ To investigate the study's objectives, data obtained in this standard follow-up routine will be recorded prospectively.

\section{Assessments}

This study will use diagnostic and outcome measures that are recorded as part of the usual clinical practice, supplemented by several questionnaires, all specified below. Outcomes are collected from the standardised feedback letter sent by physical therapists (see online supplementary file 1) and the patient's hospital electronic health record. Table 1 provides an overview of all study assessments.

\section{Baseline patient characteristics}

Participants' sociodemographic data (age, sex), medical history and comorbidity (chronic obstructive pulmonary disease, lower-extremity musculoskeletal disease, diabetes mellitus, dyslipidaemia, hypertension, kidney disease, cerebrovascular disease, ischaemic heart disease, 
Table 1 Study timeline and assessments

\begin{tabular}{|c|c|c|c|c|c|c|}
\hline & $\begin{array}{l}\text { Baseline } \\
T_{0}\end{array}$ & 3 monthst $_{1}$ & 6 monthst $_{2}$ & 12 monthst $_{3}$ & 2 yearst $_{4}$ & 5 yearst $_{5}$ \\
\hline \multicolumn{7}{|l|}{ Baseline characteristics } \\
\hline Sociodemographic data & $\mathrm{x}$ & & & & & \\
\hline Cardiovascular risk factors & $\mathrm{x}$ & & & & & \\
\hline Comorbidity & $\mathrm{x}$ & & & & & \\
\hline Medical history & $x$ & & & & & \\
\hline Prior vascular interventions & $\mathrm{x}$ & & & & & \\
\hline Vascular laboratory assessment & $x$ & & & & & \\
\hline Vascular imaging (DUS/CTA/MRA) & $X^{*}$ & $X^{*}$ & & & & \\
\hline \multicolumn{7}{|l|}{ Outcome measures } \\
\hline Treadmill test $†$ & $\mathrm{x}$ & $x$ & $x$ & $\mathrm{x}$ & & \\
\hline 6MWT† & $\mathrm{x}$ & $x$ & $\mathrm{x}$ & $\mathrm{x}$ & & \\
\hline WHOQoL-BREF & $\mathrm{x}$ & $x$ & $\mathrm{x}$ & $\mathrm{x}$ & & \\
\hline Barrier self-efficacy scale & $x$ & $x$ & $x$ & $\mathrm{x}$ & & \\
\hline VascuQoL-6 & $\mathrm{x}$ & $x$ & $\mathrm{x}$ & $\mathrm{x}$ & $\mathrm{x}$ & $\mathrm{x}$ \\
\hline Smoking status & $x$ & $x$ & $x$ & $\mathrm{x}$ & $x$ & $x$ \\
\hline Attainment of treatment goal & & $\mathrm{x}$ & $\mathrm{x}$ & $\mathrm{x}$ & $\mathrm{X}$ & $x$ \\
\hline Freedom from vascular intervention & & $x$ & $\mathrm{x}$ & $x$ & $x$ & $\mathrm{x}$ \\
\hline Major adverse cardiovascular events & & $\mathrm{x}$ & $\mathrm{x}$ & $\mathrm{x}$ & $x$ & $\mathrm{x}$ \\
\hline \multicolumn{7}{|l|}{ Personality traits } \\
\hline Big Five Inventory & $x$ & & & & & \\
\hline HADS & $\mathrm{x}$ & & & & & \\
\hline Brief Self-Control Score & $x$ & & & & & \\
\hline Life Orientation Test-Revised & $x$ & & & & & \\
\hline Barrier Self-Efficacy Scale & $\mathrm{X}$ & $\mathrm{X}$ & $\mathrm{x}$ & $\mathrm{X}$ & & \\
\hline
\end{tabular}

*Imaging is eligible when performed $\leq 3$ months before or after inclusion.

†The treadmill test and 6MWT are performed on different days, thus two visits are necessary per time point to collect all outcome measures. 6MWT, 6 min walk test; CTA, CT angiography; DUS, duplex ultrasound scanning; HADS, Hospital Anxiety and Depression Scale; IC, intermittent claudication; MRA, magnetic resonance angiography; VascuQoL-6, Vascular Quality of Life Questionnaire-6; WHOQoL-BREF, WHO Quality of Life Questionnaire-Bref.

heart failure), cardiovascular risk factors (body mass index (BMI), smoking status), prior (cardio-)vascular interventions (coronary artery bypass grafting, percutaneous coronary intervention, open or endovascular abdominal aortic aneurysm repair, lower-extremity revascularisation) and prior SET will be extracted from the electronic health records of the hospital and/or physical therapist.

Vascular imaging and laboratory assessment

Resting and postexercise ankle-brachial index (ABI) determinations in both legs are performed by trained vascular technicians in all participating centres using handheld Doppler instruments, as part of the routine work-up. The ABI is defined as the ratio between the highest systolic pressure of the dorsal pedal or posterior tibial artery, and the highest of the left or right brachial pressure. To determine the anatomic location and extent of atherosclerotic disease, the ELECT Registry uses the preferred vascular imaging modality of the treating vascular surgeon, performed $\leq 3$ months before or after inclusion.

In case of magnetic resonance angiography (MRA) or CT angiography (CTA), the scan is evaluated and interpreted by experienced radiologists in the participating centres as per usual care. A stenosis of $>50 \%$ on MRA or CTA is considered significant. The reported accuracy of CTA and MRA to detect significant arterial stenosis in PAD is $>90 \% .^{1}$ Duplex ultrasound scanning (DUS) is carried out by accredited vascular technicians to determine the location, extent and severity of the atherosclerotic lesions. A lesion is considered significant if either a peak systolic velocity ratio of $\geq 2.5$, or an end-diastolic velocity of $\geq 0.6 \mathrm{~m} / \mathrm{s}$ is observed, or if an occlusion is visualised (no flow). The reported sensitivity and specificity of DUS in patients with PAD to detect significant arterial stenoses is over $80 \%$ and $90 \%$, respectively. ${ }^{1}$ 
To categorise all participants according to anatomic location a team of three physicians (one vascular surgeon, two PhD candidates) will independently assess the DUS reports and readings and/or MRA or CTA scans and radiologist reports. Participants will be divided into four groups:

1. Aortoiliac lesions, containing patients with significant stenoses or occlusions in the common iliac artery, external iliac artery and/or internal iliac artery.

2. Femoropopliteal lesions, containing patients with significant stenoses or occlusions in the common femoral artery, superficial femoral artery and/or popliteal artery.

3. Multilevel disease, containing patients with significant stenoses at both the aortoiliac and femoropopliteal level.

4. Rest group, containing patients with no significant stenoses in the aortoiliac and femoropopliteal tract. Notably, undetected infrageniculate disease distally from the area scanned with DUS may exist in this group. This category is expected to contain some 5\% of patients meeting the eligibility criteria based on a retrospective exploratory analysis of a consecutive cohort of patients from the vascular surgery outpatient clinic in the initiating centre.

The same three physicians will also assign a Transatlantic Inter-Society Consensus for the Management of PAD (TASC) classification for each arterial trajectory with a significant lesion. Notably, the inter-observer agreement for rating TASC classifications is poor. ${ }^{20} 21$ Thus, disagreement is solved by discussion and consultation of a fourth observer (vascular surgeon).

\section{Walking performance \\ Treadmill-measured walking distance}

The maximum walking distance (MWD) and functional walking distance (FWD) will be recorded for each patient at baseline, 3, 6 and 12 months follow-up, using a standardised treadmill test. The MWD is defined as the walking distance where intolerable claudication pain forces a patient to stop. An alternative term for MWD is 'absolute claudication distance'. The FWD is defined as the distance at which the patient prefers to stop walking because of pain. Notably, this is a different measure from the 'pain-free walking distance' or 'initial claudication distance' commonly used in PAD literature. The FWD was previously found to be reliable (intraclass correlation coefficient 0.959 ) and probably a better reflection of functional impairment compared with the initial claudication distance. ${ }^{22}$ Walking distances are determined by a standardised progressive treadmill test (ie, Gardner-Skinner protocol ${ }^{23}$ ) with a constant speed of $3.2 \mathrm{~km} /$ hour starting with $0 \%$ incline, increasing every $2 \mathrm{~min}$ by $2 \%$. The maximum incline is $10 \%$, and the maximum duration of the test is $30 \mathrm{~min}$, resulting in a maximum distance of $1600 \mathrm{~m}$. The test protocol is advised by the Dutch physical therapy guidelines. ${ }^{19}$ ClaudicatioNet therapists are taught to offer verbal encouragement and coaching during testing, but this was not standardised in this study.

\section{6-Minute walk test}

To assess walking performance in a setting more resembling daily life, participants will perform the 6MWT at baseline, 3, 6 and 12 months follow-up. Physical therapists are instructed to perform the treadmill test and 6MWT on different days. The 6MWT records the total distance an individual is able to walk over a total of $6 \mathrm{~min}$ on a hard, flat surface. Participants traverse back and forth along a marked walkway. They are allowed to self-pace and rest as needed, while timing continues up to $6 \mathrm{~min}$. The 6MWT has been validated, shown to be responsive to treatment, and is predictive of mortality and mobility loss in a PAD population. ${ }^{24}$

\section{Quality of life}

Two questionnaires are used in the ELECT Registry to investigate the effect of treatment on QoL. The VascuQoL-6 is a valid and responsive instrument for the assessment of health-related QoL in PAD. ${ }^{25}$ It is a shortened version of the VascuQoL-25 and contains questions relating to the patient's activities, symptoms, pain and emotional and social well-being. Answers are recorded on a 4-point scale and added up, resulting in a total score between 6 and 24. Higher scores indicate better health-related QoL. The ELECT Registry uses the Dutch version of the VascuQoL-6.

The WHOQoL questionnaire provides a generic assessment of QoL. ${ }^{26}{ }^{27}$ The abbreviated version of this tool (WHOQoL-BREF) is used in this registry and was previously found to be valid and reliable. ${ }^{27}$ It contains questions on four domains, each scored on a 5-point Likert scale: physical health, psychological health, social relationships and environment. Two additional questions assess the subject's overall QoL and general health. Domain scores are obtained by averaging the scores from the individual questions and subsequent rescaling to a 0-100 scale, where higher scores represent a better QoL.

\section{Success of conservative management}

Arguably, a successful conservative treatment eliminates the need for invasive therapies. Thus, success of SET is objectified by freedom from vascular interventions, defined as either PTA (with or without stent), bypass, endarterectomy or major amputation of the lower extremities at 6 months, and 1, 2 and 5 years, follow-up.

The decision to intervene is influenced by the risk-benefit ratio of the patient's lesion and general health status, as well as the preferences of the treating vascular surgeon and patient. Thus, freedom from intervention is only a partial reflection of treatment success. To better capture patient satisfaction with treatment the attainment of the main treatment goal is recorded. At the start of each SET programme each patient determines the main treatment goal in conjunction with his or her physical therapist. This is recorded in an open text field at the start of treatment, 
with no restrictions with regard to the domain or measure it applies to. Satisfactory achievement of this goal ('yes' or 'no') and the (im) probability of further improvement with SET ('yes' or 'no') are followed-up at 3, 6 and 12 months and indicated in the standard feedback letter to the vascular surgeon (see online supplementary file 1 ).

\section{Cardiovascular mortality and morbidity}

The incidence of major adverse cardiovascular events (MACE) at 1, 2 and 5 years follow-up. Events considered are atherosclerotic cardiovascular death, non-fatal myocardial infarction and non-fatal stroke (three-component MACE), recorded from hospital records.

\section{Psychological assessments}

The five self-report questionnaires described below assess personality traits, emotional symptoms and barrier self-efficacy. Missing data, if not completely at random, will be imputed by means of multiple imputation methodology to minimise bias. ${ }^{28}$

\section{Big Five Inventory}

The Big Five Inventory (BFI) ${ }^{29}$ consists of 44 items regarding statements of characteristics associated with five personality traits, which are openness to experiences, agreeableness, extraversion, neuroticism and conscientiousness. This study will focus on the last three traits. Every item starts with "I see myself as someone who..." and items are rated on a 5-point Likert scale (1=disagree strongly, $5=$ agree strongly). The BFI has a high test-retest reliability with intraclass correlation coefficients ranging from 0.93 to $0.96 .^{30}$ A validated Dutch translation ${ }^{31}$ is used in this study, with satisfactory reliability for measuring the individual traits, with Cronbach's $\alpha$ ranging among traits from 0.73 to 0.86 ( $\alpha>0.7$ is a sign of good reliability). The scores will be analysed as continuous and as categorical variables. Because there are no official cut-off scores available, the median scores from the current sample will be used as cut-off points to translate scores into low and high on a specific trait ('low'<median $\geq$ 'high').

\section{Hospital Anxiety and Depression Scale}

The Hospital Anxiety and Depression Scale (HADS) is a 14-item self-report screening scale which is used to indicate the possible presence of anxiety and/or depressive symptoms. ${ }^{32}$ The scale includes seven items on anxiety and seven items on depression, both with a score ranging from 0 to 21 . The total score is classified into no anxiety or depression disorder $(\leq 7)$, possible disorder (8-10) and probable disorder $(\geq 11)$. The Dutch translation of the HADS ${ }^{33}$ used in the ELECT Registry is validated in multiple populations. The internal consistency (Cronbach's $\alpha$ ranged from 0.71 to 0.90 ) and the test-retest stability (Pearson's correlation coefficient $=0.91$ ) are high.

\section{Brief self-control score}

The brief self-control score ${ }^{34}$ is used to determine self-control in which a higher score is associated with higher self-control. It consists of 13 items rated on a 5-point
Likert scale ( $1=$ disagree strongly, $5=$ agree strongly) with a maximum score of 54 . This brief scale has a good test-retest reliability with a coefficient of 0.87 . A validated Dutch translation $^{35}$ with high internal consistency (Cronbach's $\alpha=0.80$ ) will be used.

\section{Life orientation test-revised}

The life orientation test-revised ${ }^{36}$ measures optimism by means of 10 items, including four 'filler' items which do not contribute to the total score. The items are rated on a 5-point Likert scale ( $0=$ disagree strongly, $4=$ agree strongly). The total score ranges between 0 and 24 and a higher total score is associated with a higher level of optimism. This revised test has a satisfactory test-retest reliability of $0.60-0.68$. The validated Dutch translation, ${ }^{37}$ with moderate-to-high internal consistency (Cronbach's $\alpha$ ranging from 0.58 to 0.80 ), is used.

\section{Barrier self-efficacy scale}

The barrier self-efficacy scale (BSES) $)^{38}$ consists of 13 items describing the possibility that one would exercise despite the presence of possible barriers, such as bad weather or lack of interest. The inventors of the BSES determined the barriers by attributive analysis of participants' arguments for discontinuing exercise programmes. Participants indicate their degree of confidence for each item on a $0 \%$ (no confidence at all) to $100 \%$ (complete confidence) scale. The mean percentage of all items comprises the total score, in which a higher score is associated with higher barrier self-efficacy. This study uses a validated Dutch translation ${ }^{39}$ in which one question ("My work schedule conflicted with my exercise session") is left out because this would not be relevant to the majority of our study population. The internal consistency of the Dutch scale is high (Cronbach's $\alpha=0.84$ ) and has a satisfactory test-retest stability with an intraclass correlation coefficient of 0.63 .

\section{Sample size}

We hypothesise that there will be no clinically relevant difference in changes in maximum walking distance between subjects with aortoiliac and femoropopliteal disease after 6 months. Analogous to previous trials in this population, we would consider a mean difference of $150 \mathrm{~m}$ change in walking distance between groups to be clinically relevant $(\mathrm{SD}$ of $300 \mathrm{~m}) .{ }^{40}{ }^{41}$ To exclude a difference in means of $150 \mathrm{~m}$ with an $\alpha$ of 0.01 , a power of 0.80 ( $\beta$ of 0.2 ), 96 patients are needed per arterial disease level group. The ELECT Registry primarily aims to compare patients with single-level disease (aortoiliac vs femoropopliteal). Based on a retrospective analysis of consecutive patients with IC in our hospital, this will be the case in some $75 \%$ of the patients. Thus, for the primary analysis the required sample size amounts to a total of 256 patients $((1 / 0.75) \times(96+96))$. Assuming a conservative drop-out rate of $20 \%, 320$ subjects are required to investigate the primary objective. 


\section{Statistical analysis}

Categorical variables at baseline will be presented as numbers with percentages. Continuous variables as means \pm SD if normally distributed, or as medians with interquartile ranges in case of a skewed distribution. Participants will be divided into four groups based on anatomical characteristics of disease, as apparent on the preferential imaging modality of the participating centre (either DUS, CTA or MRA). Groups are defined as follows: aortoiliac disease, femoropopliteal disease, multilevel disease or rest group. The primary comparison of interest is aortoiliac versus femoropopliteal disease.

Changes in walking distances (treadmill tests, 6MWT) and QoL (VascuQoL-6, WHOQoL-BREF) will be compared between groups, after multivariate adjustment. Adjustment of these measures for possible confounding variables will be performed using a general linear model with anatomical group as the independent variable. Covariates used for this adjustment are selected using univariate analysis (inclusion criteria $\mathrm{p}<0.2$ ). A stringent significance level of 0.01 will be used to account for multiple comparisons. Freedom from revascularisation, freedom from adverse cardiovascular disease events and attainment of the main treatment goal between groups will be compared using Kaplan-Meier survival curves and log rank tests. Cox proportional hazard analysis will be performed to adjust for the above-mentioned possible confounders.

Analysis for the remainder of secondary objectives is performed on the overall population (regardless of lesion location). Multivariable logistic regression will be used to determine the impact of several patient characteristics (eg, age, sex, extent of atherosclerotic disease, comorbidity, history of cardiovascular disease and previous lower-extremity interventions) on success of conservative management. Associations between baseline personality traits and SET-related outcomes (walking distance, 6MWT, QoL and freedom from interventions), defined as change-from-baseline scores at follow-up, will be determined. For categorised BFI traits this will be analysed with unpaired t-tests (or Mann-Whitney $\mathrm{U}$ test in case of non-normal distribution) for continuous outcomes and $\mathrm{X}^{2}$ or Fisher's exact tests for categorical outcomes. Multiple linear regression is performed with personality traits as independent variables and the SET-related outcomes as dependent variables. A hierarchical series of three models with increasing covariate adjustment will be used. In model I, age and sex are included as covariates. In model II, secondary invasive treatment (if applicable) is added. Finally, in model III, the HADS score is added in order to correct for possible symptoms of anxiety and/ or depression. To analyse the change in self-efficacy at 3 , 6 and 12 months follow-up, a linear mixed model will be used.

\section{Data storage and retention}

All data will be handled in accordance with local regulations and privacy laws in an anonymised dataset. Physical data will be anonymised and stored accessible only by the research team, digital data will be secured using dedicated data management software 'Research Manager' (de Research Manager, Deventer, the Netherlands). After the last participant's final follow-up moment, all data will be stored for a minimum of 15 years.

\section{Ethics and dissemination}

This trial does not hamper routine vascular surgery or physical therapy treatment for the participants. Furthermore, it mainly records diagnostic and outcome measures that are performed as part of the usual clinical routine, supplemented by several short, non-intrusive questionnaires. Therefore, the ELECT Registry has been exempted from formal medical ethical approval by the Medical Research Ethics Committees United 'MEC-U' (reference number W17.071). Nonetheless, privacy laws require that each subject must authorise the treating physician(s), therapists and institutions to release their medical information. Each subject must therefore sign a patient informed consent form before any data can be sent to the coordinating centre. The results are intended for publication in peer-reviewed journals and for presentation to stakeholders nationally and internationally.

\section{Patients and public involvement}

Patients and public were not explicitly involved in the design or conduct of this study. All participants will be informed of the results of the ELECT Registry through post or email.

\section{DISCUSSION}

The ELECT Registry will link anatomical characteristics of atherosclerotic disease to the functional and clinical outcomes of a SET-first management strategy in IC. This prospective observational study will thus primarily offer insight in the effect of lesion location on the outcomes of SET. It will produce novel results as most previously published research on SET as primary treatment either does not specify the location of the stenosis, does not include all lesions in its study design or does not include functional outcome measures. By including a diverse range of vascular surgery centres throughout the Netherlands, the study will feature a heterogenous patient population closely resembling real-world practice.

The dataset from this study will secondarily be used to identify predictors of treatment outcome from various functional and clinical variables. Previously published research aimed to investigate the effect of various patient characteristics on outcomes of conservative management in IC. ${ }^{42-44}$ However, this is the first study to include information on extent and location of PAD in such analysis. Moreover, the ELECT Registry will include a multitude of functional and clinical outcomes at both baseline and follow-up. The results will facilitate the development of a management strategy more tailored to the individual patient with IC. 
The ELECT Registry is an observational study and thus prone to several validity issues. Mainly, included patients in the different lesion location groups are expected to differ regarding several clinical characteristics. Indeed, van Zitteren $e t a l^{45}$ and Aboyans $e t a t^{46}$ previously found differences in smoking status, diabetes mellitus, BMI and ABI between proximal and distal disease. The influence of these potential confounders is addressed in the study protocol by applying multivariate regression analysis. Despite this covariate adjustment, unmeasured confounding could influence the results. For instance, intensity of exercise during SET sessions is not recorded, nor daily life physical activity levels of patients. Furthermore, DUS is a non-invasive and valid tool to assess location and extent of stenosis in PAD, but has its limitations. Namely, visualisation of the iliac vessels can be limited due to body habitus and/or bowel gas, possibly introducing bias in the study design.

\section{CONCLUSION}

The ELECT Registry, a multicentre prospective cohort study, will produce a dataset linking the functional and clinical outcomes of SET in IC to the location and extent of the atherosclerotic lesions. The results from this real-world cohort will inform clinical practice, working towards a more tailored management of IC patients.

\section{Author affiliations}

${ }^{1}$ Department of Vascular Surgery, Catharina Hospital, Eindhoven, The Netherlands ${ }^{2}$ CAPHRI Research School, Maastricht University Medical Centre, Maastricht, The Netherlands

${ }^{3}$ Department of Vascular Surgery, Amphia Hospital, Breda, The Netherlands ${ }^{4}$ Department of Vascular Surgery, Elisabeth Twee Steden Hospital, Tilburg, The Netherlands

${ }^{5}$ Department of Vascular Surgery, Medisch Spectrum Twente, Enschede, The Netherlands

${ }^{6}$ Department of Vascular Surgery, Rijnstate Hospital, Arnhem, The Netherlands ${ }^{7}$ Department of Vascular Surgery, VieCuri Medical Centre, Venlo, The Netherlands ${ }^{8}$ Department of Vascular Surgery, University Medical Centre Utrecht, Utrecht, The Netherlands

${ }^{9}$ Department of Vascular Surgery, Albert Schweitzer Hospital, Dordrecht, The Netherlands

${ }^{10}$ Department of Medical and Clinical Psychology, Center of Research on

Psychological and Somatic Disorders (CoRPS), Tilburg, The Netherlands

${ }^{11}$ Department of Vascular Surgery, Erasmus Medical Centre, Rotterdam, The Netherlands

${ }^{12}$ Department of Vascular Surgery, Amsterdam University Medical Centres, Amsterdam, The Netherlands

${ }^{13}$ Department of Vascular Surgery, Máxima Medical Centre, Veldhoven, The Netherlands

Contributors MMLvdH, JAWT, MRMS designed the overall study and drafted the manuscript. SCPJ, IN, EVR designed and planned the assessment of personality traits and self-efficacy. MMLvdH, AS, LvdL, PWHEV, EMW, J-WL, J-WME, ESvH, MAL, MJWK, MRMS, JAWT coordinated the planning and conduct of the study in their respective centre. All authors have contributed to the refinement of the study protocol and revision of the manuscript. All authors have read and approved the final manuscript.

Funding The authors have not declared a specific grant for this research from any funding agency in the public, commercial or not-for-profit sectors.

Competing interests None declared.

Patient consent for publication Not required.
Provenance and peer review Not commissioned; externally peer reviewed.

Open access This is an open access article distributed in accordance with the Creative Commons Attribution Non Commercial (CC BY-NC 4.0) license, which permits others to distribute, remix, adapt, build upon this work non-commercially, and license their derivative works on different terms, provided the original work is properly cited, appropriate credit is given, any changes made indicated, and the use is non-commercial. See: http://creativecommons.org/licenses/by-nc/4.0/.

\section{REFERENCES}

1. Conte MS, Pomposelli FB, Clair DG, et al. Society for Vascular Surgery practice guidelines for atherosclerotic occlusive disease of the lower extremities: management of asymptomatic disease and claudication. J Vasc Surg 2015;61:2S-41.

2. Frans FA, Bipat S, Reekers JA, et al. Systematic review of exercise training or percutaneous transluminal angioplasty for intermittent claudication. Br J Surg 2012;99:16-28.

3. Murphy TP, Cutlip DE, Regensteiner JG, et al. Supervised exercise versus primary stenting for claudication resulting from aortoiliac peripheral artery disease: six-month outcomes from the claudication: exercise versus endoluminal revascularization (CLEVER) study. Circulation 2012;125:130-9.

4. Spronk S, Bosch JL, den Hoed PT, et al. Intermittent claudication: clinical effectiveness of endovascular revascularization versus supervised hospital-based exercise training--randomized controlled trial. Radiology 2009;250:586-95.

5. Fakhry F, Rouwet EV, den Hoed PT, et al. Long-term clinical effectiveness of supervised exercise therapy versus endovascular revascularization for intermittent claudication from a randomized clinical trial. Br J Surg 2013;100:1164-71.

6. Nordanstig J, Gelin J, Hensäter M, et al. Walking performance and health-related quality of life after surgical or endovascular invasive versus non-invasive treatment for intermittent claudication-a prospective randomised trial. Eur J Vasc Endovasc Surg 2011;42:220-7.

7. Mazari FA, Khan JA, Carradice D, et al. Randomized clinical trial of percutaneous transluminal angioplasty, supervised exercise and combined treatment for intermittent claudication due to femoropopliteal arterial disease. Br J Surg 2012;99:39-48.

8. Fakhry F, Fokkenrood HJ, Spronk S, et al. Endovascular revascularisation versus conservative management for intermittent claudication. Cochrane Database Syst Rev 2018;3:CD010512.

9. Gommans LN, Fokkenrood HJ, van Dalen HC, et al. Safety of supervised exercise therapy in patients with intermittent claudication. $J$ Vasc Surg 2015;61:512-8.

10. van den Houten MM, Lauret GJ, Fakhry F, et al. Cost-effectiveness of supervised exercise therapy compared with endovascular revascularization for intermittent claudication. Br J Surg 2016;103:1616-25.

11. Hageman D, Fokkenrood HJP, Essers PPM, et al. Improved adherence to a stepped-care model reduces costs of intermittent claudication treatment in The Netherlands. Eur J Vasc Endovasc Surg 2017;54:51-7.

12. Makris GC, Lattimer CR, Lavida A, et al. Availability of supervised exercise programs and the role of structured home-based exercise in peripheral arterial disease. Eur J Vasc Endovasc Surg 2012;44:569-75.

13. Gommans LN, Teijink JA. Attitudes to supervised exercise therapy. $\mathrm{Br}$ J Surg 2015;102:1153-5.

14. Shalhoub J, Hamish M, Davies AH. Supervised exercise for intermittent claudication - an under-utilised tool. Ann R Coll Surg Engl 2009;91:473-6.

15. Hageman D, van den Houten MM, Spruijt S, et al. Supervised exercise therapy: it does work, but how to set up a program? J Cardiovasc Surg 2017;58:305-12.

16. van Zitteren $\mathrm{M}$, Vriens $\mathrm{PW}$, Burger $\mathrm{DH}$, et al. Determinants of invasive treatment in lower extremity peripheral arterial disease. J Vasc Surg 2014;59:400-8.

17. Frans FA, Bipat S, Reekers JA, et al. SUPERvised exercise therapy or immediate PTA for intermittent claudication in patients with an iliac artery obstruction--a multicentre randomised controlled trial; SUPER study design and rationale. Eur J Vasc Endovasc Surg 2012;43:466-71.

18. Greenhalgh RM, Belch JJ, Brown LC, et al. The adjuvant benefit of angioplasty in patients with mild to moderate intermittent claudication (MIMIC) managed by supervised exercise, smoking cessation advice and best medical therapy: results from two 
randomised trials for stenotic femoropopliteal and aortoiliac arterial disease. Eur J Vasc Endovasc Surg 2008:36:680-8.

19. Merry AHH, Teijink JAW, Jongert MWA, et al. KNGF-Richtlijn Symptomatisch perifeer arterieel vaatlijden [In Dutch], 2017.

20. Wu TY, Giesler G, Herscu G, et al. Agreement among observers in the assignment of transatlantic inter-society consensus classification and runoff score. J Vasc Surg 2013;58:1254-8.

21. Kukkonen T, Korhonen M, Halmesmäki K, et al. Poor inter-observer agreement on the TASC II classification of femoropopliteal lesions. Eur J Vasc Endovasc Surg 2010;39:220-4.

22. Kruidenier LM, Nicolaï SP, Willigendael EM, et al. Functional claudication distance: a reliable and valid measurement to assess functional limitation in patients with intermittent claudication. BMC Cardiovasc Disord 2009;9:9:9.

23. Gardner AW, Skinner JS, Cantwell BW, et al. Progressive vs singlestage treadmill tests for evaluation of claudication. Med Sci Sports Exerc 1991;23:402???408-8.

24. McDermott MM, Guralnik JM, Criqui MH, et al. Six-minute walk is a better outcome measure than treadmill walking tests in therapeutic trials of patients with peripheral artery disease. Circulation 2014:130:61-8

25. Larsen ASF, Reiersen AT, Jacobsen MB, et al. Validation of the Vascular quality of life questionnaire - 6 for clinical use in patients with lower limb peripheral arterial disease. Health Qual Life Outcomes 2017;15:184.

26. Öztürk Ç, te Slaa A, Dolmans DE, et al. Quality of life in perspective to treatment of postoperative edema after peripheral bypass surgery. Ann Vasc Surg 2012;26:373-82.

27. Development of the world health organization whoqol-bref quality of life assessment. The WHOQOL Group. Psychol Med 1998;28:551-8.

28. van der Heijden GJ, Donders AR, Stijnen T, et al. Imputation of missing values is superior to complete case analysis and the missing-indicator method in multivariable diagnostic research: a clinical example. J Clin Epidemiol 2006:59:1102-9.

29. John OP, Naumann LP, Soto CJ. Paradigm shift to the integrative Big Five trait taxonomy: History, measurement, and conceptual issues. In: John OP, Robins RW, Pervin LA, eds. Handbook of personality: Theory and research. New York, NY: Guilford Press, 2008:114-58.

30. Arterberry BJ, Martens MP, Cadigan JM, et al. Application of generalizability theory to the big five inventory. Pers Individ Dif 2014;69:98-103.

31. Denissen JJ, Geenen R, van Aken MA, et al. Development and validation of a Dutch translation of the Big Five Inventory (BFI). J Pers Assess 2008;90:152-7.

32. Snaith RP, Zigmond AS. The hospital anxiety and depression scale. Br Med J 1986;292:344.

33. Spinhoven P, Ormel J, Sloekers PP, et al. A validation study of the Hospital Anxiety and Depression Scale (HADS) in different groups of Dutch subjects. Psychol Med 1997;27:363-70.
34. Tangney JP, Baumeister RF, Boone AL. High self-control predicts good adjustment, less pathology, better grades, and interpersonal success. J Pers 2004;72:271-324.

35. Helmerhorst M, de Vries Robbé M. Zelf-Controle Schaal en ZelfControle Schaal Observatie. Geautoriseerde Nederlandse vertaling van de Self-Control Scale (Tangney, Baumeister, \& Boone, 2004). Utrecht: Van der Hoeven Stichting, 2011.

36. Scheier MF, Carver CS, Bridges MW. Distinguishing optimism from neuroticism (and trait anxiety, self-mastery, and self-esteem): a reevaluation of the life orientation test. J Pers Soc Psychol 1994;67:1063-78.

37. Klooster PMten, Weekers AM, Eggelmeijer F, et al. Optimisme en/of pessimisme: factorstructuur van de nederlandse life orientation testrevised. Psychologie en Gezondheid 2010;38:89-100.

38. McAuley $\mathrm{E}$. The role of efficacy cognitions in the prediction of exercise behavior in middle-aged adults. J Behav Med 1992;15:65-88

39. Stevens M, Dijk AB-van, de Greef MHG, et al. A dutch translation of a questionnaire assessing self-efficacy in leisure-time physical activity. J Aging Phys Act 2001:9:223-32.

40. Fakhry F, Spronk S, van der Laan L, et al. Endovascular revascularization and supervised exercise for peripheral artery disease and intermittent claudication: a randomized clinical trial. JAMA 2015;314:1936-44.

41. Nicolaï SP, Teijink JA, Prins MH. Multicenter randomized clinical trial of supervised exercise therapy with or without feedback versus walking advice for intermittent claudication. J Vasc Surg 2010;52:348-55.

42. Gardner AW, Parker DE, Montgomery PS. Predictors of improved walking after a supervised walking exercise program in men and women with peripheral artery disease. Int $J$ Vasc Med 2016;2016:1-8.

43. Kruidenier LM, Nicolaï SP, Ten Bosch JA, et al. Predictors of walking distance after supervised exercise therapy in patients with intermittent claudication. Eur J Vasc Endovasc Surg 2009;38:449-55.

44. Dörenkamp S, Mesters I, de Bie R, et al. Patient characteristics and comorbidities influence walking distances in symptomatic peripheral arterial disease: a large one-year physiotherapy cohort study. PLoS One 2016;11:e0146828.

45. van Zitteren M, Vriens PW, Heyligers JM, et al. Self-reported symptoms on questionnaires and anatomic lesions on duplex ultrasound examinations in patients with peripheral arterial disease. $J$ Vasc Surg 2012;55:1025-34.

46. Aboyans V, Lacroix P, Criqui MH. Large and small vessels atherosclerosis: similarities and differences. Prog Cardiovasc Dis 2007;50:112-25. 